\title{
Tilt Teste no Diagnóstico Diferencial da "Epilepsia" Resistente ao Tratamento
}

\author{
Tilt Table Test in the Differential Diagnosis of Refractory "Epilepsy" \\ Renata Rodrigues Teixeira de Castro e Antonio Claudio Lucas da Nóbrega \\ Hospital Pró-Cardíaco e Universidade Federal Fluminense, Rio de Janeiro, RJ
}

A epilepsia é uma das causas mais freqüentes de distúrbios neurológicos em adultos jovens. Relatamos um caso em que uma paciente conviveu durante doze anos com o diagnóstico de epilepsia resistente ao tratamento, quando, na verdade, a causa de seus sintomas pôde ser encontrada com a realização do teste de inclinação (tilt teste). O cardiologista deve estar alerta para o possível diagnóstico de síncope neurocardiogênica em pacientes previamente diagnosticados como portadores de epilepsia, especialmente naqueles com difícil controle terapêutico.

Epilepsia é um termo que engloba um grupo de condições crônicas cuja principal manifestação clínica é a ocorrência de convulsões epiléticas - episódios súbitos e em geral espontâneos de alteração da consciência e movimentos involuntários ${ }^{1}$. A epilepsia é uma das causas mais freqüentes de distúrbios neurológicos em adultos jovens, acometendo cerca de cinqüenta milhões de pessoas em todo o mundo ${ }^{2,3}$. A prevalência da epilepsia na Europa varia de 4,5 a sete casos a cada mil habitantes, dependendo da faixa etária considerada, sendo diagnosticados 311 mil novos casos a cada $\mathrm{ano}^{4}$. O conhecimento por parte dos médicos, da alta prevalência da epilepsia, especialmente em crianças, pode fazer com que esse diagnóstico seja estabelecido sem que se considere a possibilidade de outros diagnósticos diferenciais.

Relatamos um caso em que uma paciente conviveu durante doze anos com o diagnóstico de epilepsia resistente ao tratamento, quando, na verdade, a causa de seus sintomas pôde ser encontrada com a realização do teste de inclinação (tilt teste).

\section{Relato do caso}

Paciente do sexo feminino, vinte anos de idade, com história de cinco episódios de perda fugaz do tônus postural, sem perda da consciência (relatava que mantinha a audição durante tais episódios) desde os oito anos de idade. Todos os episódios de pré-síncope eram precedidos por mal-estar mal-definido, cansaço, turvação visual, calor e visualização de escotomas cintilantes. A paciente relatava que em nenhum dos
Epilepsy is one of the most frequent causes of neurological disorders in young adults. We report the case of a patient who lived with the diagnosis of refractory epilepsy for twelve years, when actually the cause of the symptoms could be found with the performance of a tilt table test. Cardiologists should be aware of the possible diagnosis of neurocardiogenic syncope in patients previously diagnosed with epilepsy, especially in those with difficult therapeutic control. episódios houve liberação esfincteriana, abalos musculares, mordedura de língua ou outros sinais que pudessem ser interpretados como crise convulsiva do tipo grande mal. Além disso, não havia sintomas posteriores que pudessem indicar estado de confusão pós-ictal.

Consulta neurológica após o primeiro episódio concluiu que a paciente sofria de epilepsia do tipo ausência (epilepsia do tipo pequeno mal), apesar de eletroencefalograma com resultado normal, sendo prescrita carbamazepina. Apesar do uso regular desse medicamento por período de doze anos e mantendo sempre níveis séricos terapêuticos dessa substância, a paciente apresentou quatro episódios semelhantes desde o início do tratamento. Dois deles ocorreram em posição ortostática e dois em posição sentada. Não foi possível identificar situações desencadeadoras de tais episódios, como calor, ortostatismo prolongado, estresse emocional, visibilização de sangue, dor, entre outras.

Além disso, a paciente relatava diversos episódios em que sentia todos os pródromos previamente relatados, porém sem evolução para pré-síncope e com melhora após assumir o decúbito dorsal.

Diante do diagnóstico de epilepsia resistente ao tratamento, a paciente foi submetida a nova investigação clínica. Não havia nenhuma alteração ao exame físico. Foram realizados hemograma, bioquímica, eletrocardiograma, holter de 24 horas e eletroencefalograma, sem alterações.

Procedeu-se a investigação por meio de tilt teste. O tilt teste foi realizado em ambiente silencioso, com temperatura de $26^{\circ} \mathrm{C}$ e umidade relativa do ar de $54 \%$, com monitorização eletrocardiografia contínua (ECG 98 ${ }^{\circledR}, \mathrm{HW}$,

\section{Palavras-chave}

Síncope, epilepsia, tilt teste, diagnóstico diferencial, crise convulsiva. 


\section{Relato de Caso}

Brasil) e monitorização intermitente da pressão arterial (PA) por esfigmomanômetro digital (Vital Lite ${ }^{\circledR}$, Indumed, Brasil). Após dez minutos de decúbito dorsal, foi realizada mudança passiva para posição ortostática a $70^{\circ}$, sendo essa mantida por vinte minutos. Em seguida, administraram-se 400 mcg de gliceril-trinitrato (Nitrolingual Pumpspray ${ }^{\circledR}$, G. Pohl-Boskamp $\mathrm{GmbH} \& \mathrm{Co}$, Alemanha) por via sublingual. No 40을 minuto de ortostatismo, a paciente iniciou queixa de mal-estar maldefinido, cansaço, turvação visual, calor e visualização de escotomas cintilantes. Houve intensificação dos sintomas, palidez e pré-síncope. Melhora do quadro clínico após retorno ao decúbito dorsal.

A completa reprodução dos sintomas que levaram à investigação, associada à queda abrupta dos valores de PA (queda de PA sistólica $=40 \mathrm{mmHg}$ e de PA diastólica $=$ $40 \mathrm{mmHg}$, PA mínima $=77 \times 32 \mathrm{mmHg}$ ) e da freqüência cardíaca ( $\mathrm{FC}$ mínima $=63 \mathrm{bpm}$ ) permitiram o diagnóstico de pré-síncope neurocardiogênica do tipo mista em resposta ao tilt test (fig. 1). Esse padrão de resposta ao tilt teste caracterizase pela queda abrupta dos valores de PA e FC, desde que o menor valor de FC seja menor que $90 \%$ de seu valor máximo atingido no teste (nesse caso, FC máxima $=107$ bpm; 90\% da FC máxima $=96$ bpm) e maior que $40 \mathrm{bpm}$.

Após o exame, foi suspensa a administração de carbamazepina e a paciente iniciou tratamento com fludrocortisona sendo orientada quanto às medidas comportamentais a serem tomadas para prevenção dos episódios, como uso de meia elástica de alta compressão, treinamento aeróbico e aumento da ingesta de sal e água ${ }^{5}$.

\section{Discussão}

A síncope representa um sintoma freqüente, sendo a causa de $3 \%$ a $5 \%$ dos atendimentos de emergência e $1 \%$ a $3 \%$ das internações hospitalares ${ }^{6,7}$. Sua prevalência varia de acordo com a faixa etária estudada, variando de 15\% em crianças até os dezoito anos de idade ${ }^{8}$, até $23 \%$ em idosos com mais de setenta anos de idade ${ }^{9}$.

A maior prevalência de síncope em populações adultas e a grande incidência de epilepsia em crianças muitas vezes induzem à aceitação diagnóstica de epilepsia em crianças com sintomas neurológicos (perda de consciência), sem que se prossiga em investigação mais aprofundada.

Além disso, o diagnóstico de epilepsia do tipo pequeno mal muitas vezes é clínico. Apesar de existir um padrão eletroencefalográfico típico desse modo de epilepsia, a inexistência de alterações eletroencefalógraficas em paciente com forte suspeita clínica não exclui tal diagnóstico ${ }^{10}$. As alterações eletroencefalógraficas podem, eventualmente, ser registradas apenas durante os breves momentos de perda da consciência.

O caso aqui descrito continha dados que apontavam para a possibilidade diagnóstica de síncope neurocardiogênica, como os pródromos descritos, a recuperação rápida e ausência de confusão pós-ictal. Entretanto, apesar de esses dados auxiliarem na diferenciação da epilepsia clássica, do tipo grande mal, apresentam menor valor no diagnóstico da epilepsia do tipo pequeno mal, pois essa, em geral, não é seguida por estado confusional pós-ictal.

Vale ressaltar que a presença de abalos musculares não exclui o diagnóstico de síncope. Pacientes com síncope neurocardiogênica de padrão cardioinibitório podem apresentar longos períodos de assistolia, com importante prejuízo à perfusão cerebral ${ }^{11}$. Nesses casos, o paciente pode apresentar movimentos tônicos ou tônicoclônicos, assimétricos ou generalizados. Usualmente essas "convulsões" desaparecem após o paciente ser colocado em posições que facilitem a perfusão cerebral (decúbito dorsal ou posição de Tredelemburg). Apesar de tais movimentos serem muito semelhantes às convulsões características da

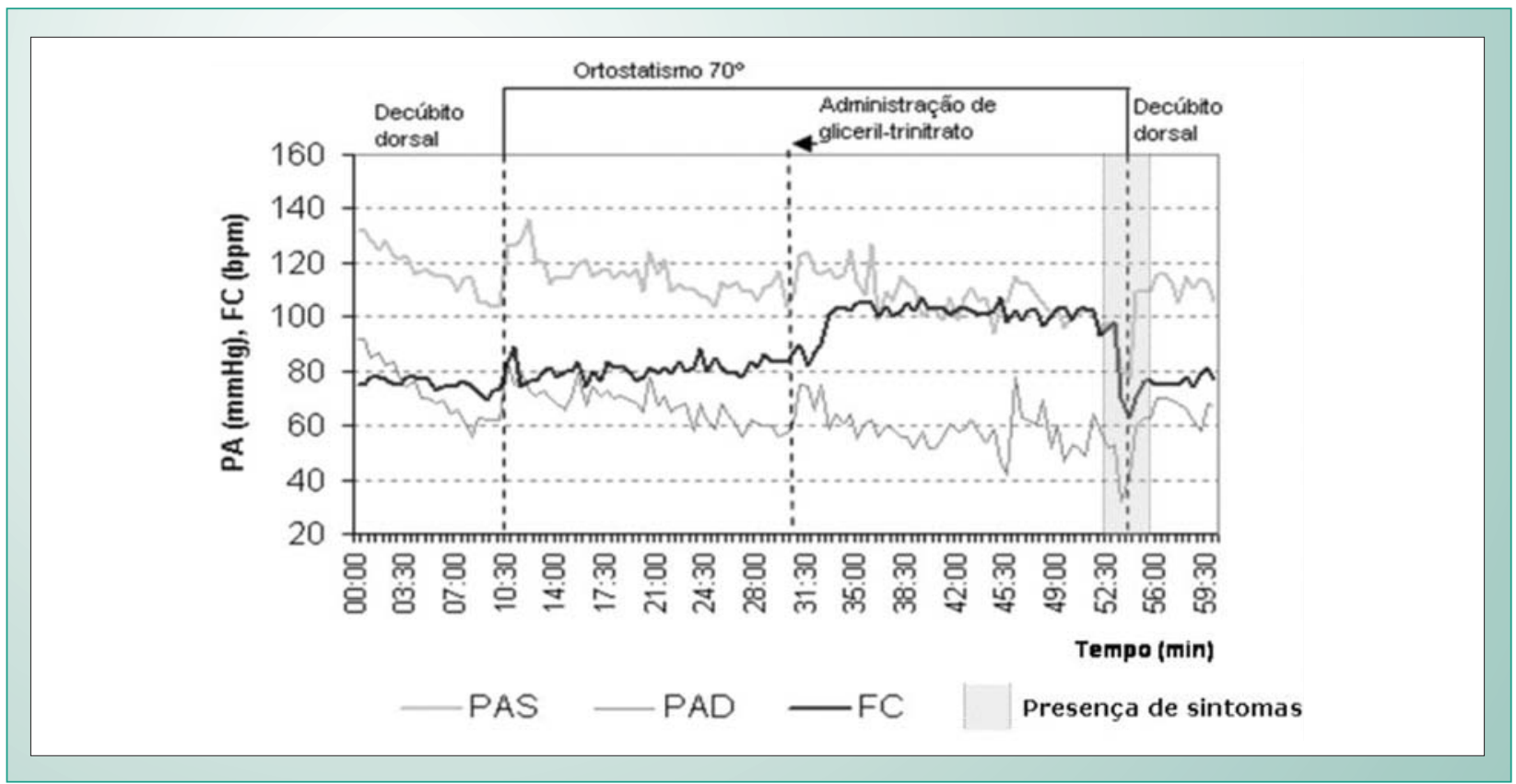

Fig. 1 - Tilt teste da paciente. PAS = pressão arterial sistólica; $P A D=$ pressão arterial diastólica; $F C=$ freqüência cardíaca. 
epilepsia, essas podem ser diferenciadas pela presença de estado confusional pós-ictal ${ }^{12}$.

O aparecimento de movimentos similares às convulsões parece ser mais freqüente em crianças. Fernandez Sanmartin e cols. ${ }^{13}$ publicaram uma casuística em que $11,6 \%$ de 216 pacientes com idade entre cinco e dezoito anos apresentaram "convulsões" durante tilt teste positivo para síncope neurocardiogênica.

Casos em que a síncope neurocardiogênica é interpretada como epilepsia tornaram-se mais comuns com a popularização do tilt teste. Em 2000, Zaidi e cols. ${ }^{14}$ avaliaram 74 pacientes com diagnóstico de epilepsia resistente ao tratamento. Quarenta e um por cento desses pacientes apresentaram tilt teste positivo. Grande parte

\section{Referências}

1. Pedley TA. The epilepsies. In: Goldman L, Bennett JC (eds). Cecil-Textbook of Medicine. 21st ed. Philadelphia: WB Saunders Company; 2000.

2. Kwan P, Brode MJ. Early identification of refractory epilepsy. N Eng J Med 2000; 342: 314-9.

3. Betting L, Kobayashi E, Montenegro MA, Min LL, Guerreiro MM, Guerreiro CAM. Tratamento da epilepsia: consenso dos especialistas Brasileiros. Arq Neuropsiquiatr 2003; 61: 1045-70.

4. Forsgren L, Beghi E, Oun A, Sillanpaa M. The epidemiology of epilepsy in Europe - a systematic review. Eur J Neurol. 2005; 12: 245-53.

5. Alboni P, Benditt D, Bergfeldt L, Blanc JJ, Bloch Thomsen PE, van Dijk JG, et al. Task Force Report- Guidelines on management (diagnosis and treatment) of syncope. Eur Heart J 2001; 22: 1256-306.

6. Day SC, Cook EF, Funkenstein H, Goldma L. Evaluation and outcome of emergency room patients with transient loss of consciousness. Am J Med $1982 ; 73: 15-23$

7. Morichetti A, Astorino G. Epidemiological and clinical findings in 697 syncope events. Minerva Medica 1998; 89: 211-20.

8. Lewis DA, Dhala A. Syncope in pediatric patient. Pediatr Clin North Am 1999; 46: 205-19. desses pacientes apresentava diagnóstico de síncope neurocardiogênica e não de epilepsia.

\section{Conclusão}

Dados epidemiológicos, como prevalência de doenças, devem ser utilizados para nortear a investigação clínica baseada em três pilares: anamnese, exame físico e exames complementares. O cardiologista deve estar alerta para o possível diagnóstico de síncope neurocardiogênica em pacientes previamente diagnosticados como portadores de epilepsia, principalmente naqueles com difícil controle terapêutico. O tilt teste é uma ferramenta simples e nãoinvasiva que pode ser útil na identificação de diagnósticos diferenciais nestes casos.
9. Lipsitz LA, Pluchino FC, Wei JY, Rowe JW. Syncope in an elderly institutionalized population: prevalence, incidence and associated risk. Q J Med 1985; 55: 45-54.

10. Aminoff MJ. Electrophysiologic studies of the central and peripheral nervous systems. IN: Braunwald E, Fauci AS, Kasper DL, Hauser SL, Longo DL, Jameson JL. (eds) Harrison's principles of internal medicine.15th ed. New York: McGraw-Hill; 2001.

11. Aminoff MJ, Scheinman MM, Griffin JC, Herre JM. Electrocerebral accompaniments of syncope associated with malignant ventricular arrhythmias. Ann Intern Med 1988; 108: 791-6.

12. Grubb BP, Gerard G, Roush K, et al. Differentiation of convulsive syncope and epilepsy with head-up tilt testing. Ann Intern Med 1991; 115: 871-6.

13. Fernandez Sanmartin M, Rodriguez Nunez A, Martinon-Torres F, Eiris Punal J, Martinon Sanchez JM. Convulsive syncope: characteristics and reproducibility using the tilt test. An Pediatr (Barc) 2001; 59: 441-7.

14. Zaidi A, Clough P, Copper P, Scheepers B, Fitzpatrick AP. Misdiagnosis of epilepsy: many seizure-like attacks have a cardiovascular cause. J Am Col Cardiol 2000; 36: 181-4. 